\title{
Improving outcomes post-radical prostatectomy: nerve-sparing status and urinary continence
}

\author{
Christopher G. Morash, MD, FRCSC, ${ }^{*}$ Ilias Cagiannos, MD, FRCSC; ${ }^{*}$ Anthony J. Bella, MD, FRCSC ${ }^{* \neq}$
}

See related article on page 465

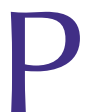

ostoperative urinary incontinence following radical prostatectomy is an increasingly important health outcome. In an era in which the burden of treatment for prostate cancer is being closely examined in terms of harm from potential overtreatment, it is important to study ways to reduce the incidence of this complication. Incontinence often results in significant stress and anxiety for patients and their families, and a significant use of resources, including incontinence products, further surgical intervention and patient and surgeon time. For most individuals, incontinence has a greater negative impact on quality of life than even erectile dysfunction. Several prior publications have looked at whether nerve sparing at radical prostatectomy contributes to continence rates, albeit with inconsistent results. ${ }^{1}$ Limitations of this literature include mostly retrospective data collection, the lack of an objective method of measuring the quality of nerve preservation itself, variable definitions of continence and its pathophysiology, lack of baseline data and a lack of objective measures of urethral and sphincter trauma intra-operatively.

In the current study, Toren and colleagues ${ }^{2}$ are to be commended for the prospective use of a validated, patientreported tool for continence outcomes, including preoperative baseline measures. However, the variable being investigated is whether nerve sparing was performed, unilateral or bilateral, and the subsequent impact on patient continence. Key data points, including nerve sparing, were obtained retrospectively based on the surgeon's description in the patient record. The surgeon's judgment may not actually correlate well with actual nerve preservation. In a study by Kaiho and colleagues, ${ }^{3}$ electrophysiological assessment of macroscopically preserved nerves were reclassified as not intact in $20 \%$ of cases, and in a third of cases the surgeon's assessment of unilateral, bilateral or nonnerve sparing was not supported by the electrophysiological assessment. Therefore, just as we require patient-reported outcomes measures, perhaps we should also require an objective metric for whether nerve preservation, unilateral or bilateral or non-nerve-sparing, was actually carried out.

Recent anatomic studies have resulted in a re-examination of the "dorsolateral neurovascular bundle" concept, as topog- raphy and nerve quantification studies confirm wide variability of periprostatic nerve distribution. ${ }^{4-6}$ Sievert and colleagues report that bilateral nerve-sparing prostatectomy for localized prostate cancer provides the possibility of preserving approximately $55 \%$ of periprostatic nerve fibres focused on the posterolateral location and $80 \%$ to $90 \%$ at the apex. ${ }^{6}$ Regardless of whether optical magnification, intraoperative nerve stimulation, or adaptations to surgical tissue handling/technique of nerve sparing are used, the actual course of nerve fibres is difficult to identify and limits optimizing clinical outcomes (both continence and erectile function) that rely upon neuronal recovery post-injury and intact nerve signaling to the sphincter or corpora. ${ }^{5}$ The endogenous response and potential for exogenous neuromodulation of the injured nerves and the interface between nerve and sphincter post-radical prostatectomy remains a physiologic "black box." While with further research we will likely attain more detailed neuroanatomical understanding, all surgical approaches inherently result in tissue disruption and patient outcomes will be best optimized by minimizing collateral damage and the development of novel neurotherapeutics. Current concepts of nerve sparing are most likely inaccurate and remain difficult to quantify. Therefore, studies relying upon a surgeon's perception of post-prostatectomy nerve status will continue to have these limitations. In essence, we currently do not have the answer to the question of whether nerve sparing or nerve sacrifice plays a role in continence outcomes.

Where does this leave us in 2010 and how do we counsel our patients? The "best" study of urinary and sexual outcomes after radical prostatectomy is yet to be reported. The ideal information would be surgeon- and patient-specific, a lofty (and difficult-to-realize) goal, and take into account prospective and accurate measures of continence status pre- and post-prostatectomy using validated metrics. This information would allow us to identify patient and surgical factors including quality and degree of nerve sparing. We also need to differentiate between underlying causes of incontinence, as anatomic and physiologic (overactive bladder) can occur separately or concurrently and likely do not have the same etiologic factors. The same step-wise approach 
can be applied to post-prostatectomy erectile dysfunction. We favour a multidisciplinary clinic approach incorporating the surgeon along with continence and erectile dysfunction specialists.

Given the contemporary knowledge gaps, and the uncertainty of whether or not nerve-sparing status influences continence preservation, in our multi-specialist clinic we do not directly address nerve preservation as it relates specifically to urine control with our patients. However, we do espouse the concept of normal tissue preservation, in that this allows for the best possible anatomic and neurophysiologic continuity. In this way, even if nerve preservation for potency is not a patient priority, and by doing so cancer control is not compromised, nerve-sparing approaches are recommended until definitive data emerge.

From the ${ }^{\star}$ Division of Urology, Department of Surgery, University of Ottawa Hospital; ${ }^{\dagger}$ Medical Director, The Ottawa Hospital Prostate Cancer Assessment Centre; ${ }^{\ddagger}$ Associate Scientist, Neuroscience, Ottawa Health Research Institute, University of Ottawa, Ottawa, ON

Competing interests: None declared.

This paper has been peer-reviewed.

\section{References}

1. Tzou DT, Dalkin BL, Christopher BA, et al. The failure of a nerve sparing template to improve urinary continence after radical prostatectomy: attention to study design. Urol Oncol 2009;27:358-62.

2. Toren $P$, Alibhai SMH, Matthew A, et al. The effect of nerve-sparing surgery on patient-reported continence post-radical prostatectomy. Can Urol Assoc J 2009;3:465-70.

3. Kaiho $\mathrm{Y}$, Nakagawa $\mathrm{H}$, Ikeda $\mathrm{Y}$, et al. Intraoperative electrophysiological confirmation of urinary continence after radical prostatectomy. J Urol 2005;173:1139.

4. Ganzer R, Blana A, Stolzenburg J-U, et al. Nerve Quantification and Computerized Panimetry to Evaluate Periprostatic Nerve Distribution - Does Size Matter? Urology 2009;74:398-403.

5. Sievert K-D, Hennenlotter J, Laible I, et al. The Periprostatic Autonomic Nerves - Bundle or Layer? Eur Urol 2008:54:1109-16

6. Sievert KD, Hennenlotter J, Laible IA, et al. The commonly performed nerve sparing total prostatectomy does not acknowledge the actual nerve courses. J Urol 2009;181:1076-81.

Correspondence: Dr. Christopher Morash, University of Ottawa, Department of Surgery, Division of Urology, The Ottawa Hospital-Civic Campus, 1053 Carling Avenue, Ottawa, ON KIY 4E9; fax: 613-761-5305; cmorash@ottawahospital.on.ca 